\title{
Sedentarismo e Síndrome Metabólica em usuários de uma Unidade de Saúde da
}

\section{Família em Salvador- BA}

\author{
Sedentarism and Metabolic Syndrome in users of a Family Health Unit in Salvador- BA \\ Sedentarismo y Síndrome Metabólico en usuarios de una Unidad de Salud de la Familia en \\ Salvador- BA
}

Julita Maria Freitas Coelho ORCID: https://orcid.org/0000-0002-9520-5177 Instituto Federal de Educação da Bahia, Brasil E-mail: julitamaria@gmail.com

Anderson Santos da Silva

ORCID: https://orcid.org/0000-0002-6601-681X

Faculdade Anísio Teixeira, Brasil

E-mail: andersonhec8@gmail.com

Eleriane Xavier dos Santos Brito

ORCID: https://orcid.org/ 0000-0003-4824-7259

Faculdade Anísio Teixeira, Brasil

E-mail: andersonhec8@gmail.com

Emily Moreira Marques

ORCID: https://orcid.org/0000-0002-5825-8608

Faculdade Anísio Teixeira, Brasil

E-mail: emilymoreiraaa@hotmail.com

Glaucia Alencar Ponte

ORCID: https://orcid.org/0000-0003-4606-3595

Secretaria Municipal de Saúde de Salvador-Ba, Brasil

E-mail: alencarglaucia@gmail.com

Érica Velasco Dias Gomes

ORCID: https://orcid.org/0000-0003-2236-9450

Universidade do Estado da Bahia, Brasil

E-mail: enfa.ericavelasco@gmail.com

Karine Gomes Lima

ORCID: https://orcid.org/0000-0001-5764-0541

Faculdade Anísio Teixeira, Brasil

E-mail: karineglima@gmail.com

Lyvia Mirelle Carneiro de França

ORCID: https://orcid.org/ 0000-0003-0844-9833

Secretaria Municipal de Saúde de Salvador-Ba, Brasil

E-mail: lyvia_franca@hotmail.com

Luiz Alberto da Silva Lima

ORCID: https://orcid.org/0000-0003-0804-2590

Universidade do Estado da Bahia, Brasil

E-mail: lalima@hotmail.com.br

Magno Conceição das Mêrces

ORCID: https://orcid.org/ 0000-0003-3493-8606

Universidade do Estado da Bahia, Brasil

E-mail: mmerces@uneb.br

Lorena Moura de Assis Sampaio

ORCID: https://orcid.org/ 0000-0003-0627-4494

Universidade Estadual de Feira de Santana, Brasil

E-mail: lorenamoura@fat.edu.br

Beatriz de Santana Fernandes

ORCID: https://orcid.org/ 0000-0001-7272-1219 Faculdade Anísio Teixeira, Brasil

E-mail: beatrizsfernandes26@gmail.com

Isabelle Matos Pinheiro

ORCID: https://orcid.org/ 0000-0001-5826-6272

Instituto Federal de Educação da Bahia, Brasil

E-mail: isabellemp@gmail.com

Lorena Ramalho Galvão

ORCID: https://orcid.org/0000-0002-4643-8994

Universidade Estadual de Feira de Santana, Brasil

E-mail: lore.galvao@hotmail.com 


\begin{abstract}
Resumo
Introdução: A síndrome metabólica representa um conjunto de distúrbios metabólicos que incluem anormalidades endócrinas e bioquímicas. Objetivo: Medir a associação entre sedentarismo e síndrome metabólica em usuários de uma Unidade de Saúde da Família em Salvador-BA Materiais e método: Foram levantados dados de um estudo prévio, do tipo transversal, com amostra de conveniência. O sedentarismo foi a variável de exposição principal e a SM foi o desfecho. Foram obtidas frequências simples e absolutas para todas as variáveis selecionadas, e Odds Ratio (OR) entre as exposições e o desfecho, assim como, um modelo final ajustado por potenciais confundidores da associação. Resultados: Dos 90 participantes, 58 eram mulheres e 22 eram homens, sendo que 36,36\% desses homens e 54,39\%

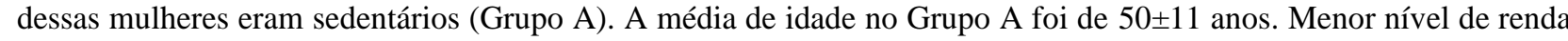
e escolaridade foi mais frequente. A prevalência de SM, segundo os critérios do NCEP-ATPIII, foi de 57,14\% entre os sedentários. No grupo com síndrome metabólica houve maior frequência de hipertensão, diabetes e alto índice de massa corporal. Não foi observada uma diferença significativa entre o sedentarismo e a síndrome metabólica, mesmo após ajustar por sexo, idade e consumo de bebida alcoólica $(\mathrm{OR}=1,02$; IC [0,42 - 2,20]. Considerações finais: A despeito de não ter sido detectada uma associação entre sedentarismo e SM, entende-se que os fatores de risco para a SM e suas alterações são bastante complexos e impactantes para a saúde como um todo.
\end{abstract}

Palavras-chave: Síndrome metabólica; Sedentarismo; Epidemiologia.

\begin{abstract}
Introduction: The metabolic syndrome represents a set of metabolic disorders that include endocrine and biochemical abnormalities. Objective: To measure the association between physical inactivity and metabolic syndrome in users of a Family Health Unit in Salvador-BA Materials and method: Data were collected from a previous cross-sectional study with a convenience sample. Sedentary lifestyle was the main exposure variable and MS was the outcome. Simple and absolute frequencies were obtained for all selected variables, and Odds Ratio (OR) between exposures and outcome, as well as a final model adjusted by potential confounders of the association. Results: Of the 90 participants, 58 were women and 22 were men, with $36.36 \%$ of these men and $54.39 \%$ of these women being sedentary (Group A). The average age in Group A was $50 \pm 11$ years. Lower income and education levels were more frequent. The prevalence of MS, according to the NCEP-ATPIII criteria, was 57.14\% among sedentary people. In the group with metabolic syndrome, there was a higher frequency of hypertension, diabetes and high body mass index. There was no significant difference between physical inactivity and metabolic syndrome, even after adjusting for sex, age and alcohol consumption (OR $=1.02$; CI $[0.42-2.20]$. Final considerations: Despite no association between sedentary lifestyle and MS has been detected, it is understood that the risk factors for MS and its changes are quite complex and impacting on health as a whole.
\end{abstract}

Key words: Metabolic syndrome; Sedentary lifestyle; Epidemiology.

\title{
Resumen
}

Introducción: El síndrome metabólico representa un conjunto de trastornos metabólicos que incluyen anomalías endocrinas y bioquímicas. Objetivo: Medir la asociación entre inactividad física y síndrome metabólico en usuarios de una Unidad de Salud de la Familia en Salvador-BA Materiales y método: Se recolectaron datos de un estudio transversal previo con una muestra de conveniencia. El estilo de vida sedentario fue la principal variable de exposición y la EM fue el resultado. Se obtuvieron frecuencias simples y absolutas para todas las variables seleccionadas, y Odds Ratio (OR) entre las exposiciones y el resultado, así como un modelo final ajustado por posibles factores de confusión de la asociación. Resultados: De los 90 participantes, 58 eran mujeres y 22 eran hombres, siendo el 36,36\% de estos hombres y el 54,39\% de estas mujeres sedentarias (Grupo A). La edad media en el grupo A fue de $50 \pm 11$ años. Los menores niveles de ingresos y educación fueron más frecuentes. La prevalencia de SM, según los criterios NCEP-ATPIII, fue del 57,14\% entre las personas sedentarias. En el grupo con síndrome metabólico, hubo mayor frecuencia de hipertensión, diabetes e índice de masa corporal elevado. No hubo diferencias significativas entre la inactividad física y el síndrome metabólico, incluso después de ajustar por sexo, edad y consumo de alcohol (OR = 1,02; IC [0,42 - 2,20]. Consideraciones finales: A pesar de no se ha detectado asociación entre el sedentarismo y la EM, se entiende que los factores de riesgo de la EM y sus cambios son bastante complejos e impactan en la salud en su conjunto.

Palabras clave: Síndrome metabólico; Estilo de vida sedentario; Epidemiología. 


\section{Introdução}

A síndrome metabólica (SM) é considerada uma epidemia mundial, e representa um transtorno complexo, com impacto socioeconômico considerável (Kassi, Pervanidou, Kaltsas \& Chrousos, 2011). Compreende um conjunto de distúrbios metabólicos que incluem anormalidades endócrinas e bioquímicas tais como: intolerância à glicose, hiperinsulinemia, resistência à insulina, hipercotisolismo, hipertrigliceridemia, HDL reduzido, que, em combinação com fatores genéticos e obesidade abdominal, representam fatores de risco para doenças cardiovasculares, diabetes mellitus tipo 2, doenças renais, hepáticas, entre outras (Bonomini, Rodella \& Rezzani, 2015; Han \& Lean, 2010).

Existem várias definições para a SM e embora a maioria das organizações internacionais e grupos de especialistas concordem que a obesidade, hipertensão, dislipidemia e tolerância anormal à glicose devam ser levadas em consideração no diagnóstico, não existe um critério padrão definido para tal (Shin et al., 2013). Também vale ressaltar que ainda não existe um mecanismo patogênico aceito mundialmente, apesar da existência de vários componentes na SM. A obesidade e a resistência à insulina em vários estudos permanecem no centro da fisiopatologia, porém uma série de outros fatores estão envolvidos na sua patogênese (Kassi et al. 2011).

Ortega et al. (2013), a partir de resultados de um estudo de coorte, chamaram atenção para a relação entre o declínio da atividade física e o aumento do sedentarismo em adolescentes estonianos e suecos de 9-15 anos, frente ao aumento do risco de obesidade e outras doenças ao longo da vida. De uma forma geral, os fatores de risco para SM parecem ser mais frequentes na fase adulta/idosa e a adoção de uma prática de exercícios físicos regulares tem sido considerada como protetor de alterações metabólicas (França; Souza \& Marques, 2017; Souza, 2010).

Nesse sentido, pontua-se que o excesso de peso é considerado o principal agravo nutricional que acomete crianças, adolescentes, adultos e idosos no Brasil, dentre os agravos relacionados a uma alimentação inadequada. Desta maneira, a obesidade além de ser uma doença importante é contabilizada na rede causal de outras DCNT (Bortolini et al., 2020). Sabe-se que são inúmeras as consequências e/ou complicações da SM. Dentre elas destacam-se a hipertensão arterial, DM e cardiopatias, as quais, dentre outras, impactam diretamente na qualidade de vida, levam a mortes prematuras e evitáveis e afetam a capacidade produtiva dos indivíduos acometidos. Além do mais, onera o Sistema Único de Saúde (SUS) em virtude do aumento da demanda de atendimentos e internações hospitalares e distribuição de medicação nas Unidades Básicas de Saúde (Nilson, Andrade, de Brito \& Oliveira, 2020; Sociedade Brasileira de Diabetes [SBD], 2017).

A referida síndrome tem uma relação direta com a ocorrência das principais causas de morte prematura na faixa etária de 30 a 69 anos, tais como desfechos cardiovasculares, diabetes, doenças renais crônicas e alguns cânceres associados à dieta inadequada (Brasil, 2018). Vale ressaltar que as doenças cardiovasculares (DCV) e o diabetes no ano de 2013 totalizaram 72,9 mortes por 100 mil habitantes no Brasil (Malta et al., 2018).

Com ampla distribuição mundial, a SM apresentou na América Latina, durante os anos de 2003 e 2006, uma prevalência relativa de $24,9 \%$, principalmente em mulheres (39,4\% versus 21,3\%), pós-menopausadas e em indivíduos com mais de 50 anos. Além disso, observaram-se nessa população maiores prevalências de dislipidemia e obesidade abdominal quando comparadas às obtidas em países desenvolvidos (Silva, Santos \& Almeida, 2017).

No Brasil, o primeiro estudo com amostra representativa da população foi realizado em 2013 e apontou uma prevalência de 8,9\% em adultos e idosos, segundo o critério de harmonização dos consensos (Ramires et al., 2018). Um estudo conduzido em um estado da região Nordeste do Brasil encontrou uma prevalência de SM de 50,7\% em adultos com Diabetes Mellitus (DM) tipo 2 segundo os critérios do NCEP ATPIII revisado. Identificou-se que ela estava associada a danos econômicos e sociais inestimáveis em decorrência, principalmente da agregação de fatores e irregularidades cardiometabólicas (Lira Neto et al., 2018). Já no município de Feira de Santana, interior do Estado da Bahia, no ano de 2015 foi estimada uma 
prevalência de mais de $60 \%$ em um grupo de usuários atendidos em um Centro de Tratamento de Diabetes e Hipertensão (Gomes Filho, et al. 2016).

A partir dos dados supracitados, reafirma-se a relevância da identificação e enfrentamento dos fatores de risco para SM, frente seus potenciais efeitos deletérios à saúde e as repercussões negativas na qualidade de vida, o que a coloca no patamar de um problema de saúde pública. Em especial o sedentarismo, dada sua capacidade de influenciar no desenvolvimento de algumas patologias metabólicas, devido ao aumento do acúmulo de tecido adiposo na composição corporal (Santos, et al., 2020; Silva, et al., 2020).

Diante do exposto, considerando a magnitude do problema e a escassez de dados acerca da SM, especialmente no Estado da Bahia, faz-se necessário a realização de estudos direcionados a esta temática. Logo, o objetivo dessa pesquisa foi medir a associação entre sedentarismo e SM em usuários de uma Unidade de Saúde da Família em Salvador/BA.

\section{Materiais e Método}

Foi conduzido um estudo de corte transversal de caráter descritivo, de natureza quantitiva (Pereira et al., 2018), que utilizou um banco de dados construído a partir de informações de prontuários e mediante aplicação de questionário, além de aferição direta de medidas antropométricas. Os participantes do estudo de base eram usuários cadastrados no Programa Hiperdia em uma Unidade de Saúde da Família (USF), em Salvador- Bahia e atendidos no período da coleta de dados. Tal programa tem como objetivo a realização do cadastramento e acompanhamento dos usuários portadores de hipertensão arterial e/ou diabetes mellitus atendidos no SUS (Brasil, 2001).

A amostra supracitada foi escolhida por conveniência e incluiu usuários com idade maior ou igual a 30 anos, que possuíam em seu prontuário registro de exames laboratoriais de triglicerídeos, HDL e glicemia de jejum, realizados nos 90 dias antecedentes ao exame clínico geral e que aceitaram participar do estudo após assinatura do Termo de Consentimento Livre e Esclarecido (TCLE). Foram excluídos aqueles usuários que possuíam uma ou mais das seguintes condições: (I) possuir infecções sistêmicas; (II) ser portadores de HIV-AIDS; e (III) estar gestante. Assim, a partir da cessão desse banco, a presente investigação levantou os dados referentes às características sociodemográficas, estilo de vida, presença de sedentarismo, além de parâmetros lipídicos e glicêmicos e condições gerais de saúde.

O diagnóstico da SM empregado se embasou em critérios preconizados pelo National Cholesterol Education Program's Adul Treatmen Panel (National Cholesterol Education Program's Adul Treatmen Panel III [NCEP-ATP III], 2001). O mesmo estabelece como necessária a presença de pelo menos três dentre cinco fatores para diagnóstico da SM, sendo esses a obesidade abdominal (circunferência abdominal $>88 \mathrm{~cm}$ para mulheres ou $>102 \mathrm{~cm}$ para homens), o aumento de triglicerídeos ( $\mathrm{TG} \geq 150 \mathrm{mg} / \mathrm{dL}$ ), a redução do HDL-col (HDL-col $<50 \mathrm{mg} / \mathrm{dL}$ para mulheres ou $<40 \mathrm{mg} / \mathrm{dL}$ para homens), a pressão arterial elevada (PA $\geq 130 / 85 \mathrm{mmHg}$ ) e a glicemia de jejum elevada ( $\geq 100 \mathrm{mg} / \mathrm{dL}$ ).

A coleta do estudo de base (Ponte et al., 2020) foi autorizada pela instituição sediadora e referendada por um Comitê de Ética em Pesquisa da Universidade Estadual de Feira de Santana (CAAE: 72539417.6.0000.0053). Ademais, todos os compromissos éticos com os pressupostos da pesquisa que envolve seres humanos foram mantidos, especialmente quanto ao sigilo e confidencialidade das informações.

A análise dos dados foi realizada a partir de frequências absolutas e relativas de todas as variáveis de interesse, e utilização de Odds Ratio (OR) entre as mesmas e a presença de SM. Para variáveis contínuas foram obtidas medidas de tendência central, média e mediana, e desvio padrão como medida de dispersão. Os testes de Qui-quadrado e Exato de Fisher foram empregados para análises bivariadas identificando possíveis associações entre as variáveis independentes e o desfecho em tela. Para testar a diferença de médias foi utilizado o teste $\mathrm{T}$ Student ou Exato de Fischer. Foi empregada análise estratificada para verificar as diferenças entre todas as exposições estudadas e SM. Foram construídas tabelas de contingência 
entre a variável dependente, dicotômica, com cada uma das variáveis independentes, de acordo com sua natureza. As OR foram obtidas com respectivos Intervalos de Confiança de 95\% (IC 95\%) e valores de p obtendo-se um modelo final ajustado por sexo, idade e consumo de bebidas alcoólicas. As análises dos dados foram procedidas com auxílio do Software de pacote estatístico STATA, versão 11.0.

\section{Resultados}

Os 90 participantes cujos dados foram levantados foram alocados em dois grupos de comparação, a partir da ausência ou presença de prática de atividade física, a saber, sedentários (Grupo A) e não sedentários (Grupo B). No geral os grupos mostraram homogeneidade em relação às variáveis estudadas. A despeito das diferenças matemáticas verificadas, somente houve diferenças estatisticamente significantes para escolaridade e IMC. Do total de participantes, $66,67 \%$ eram sedentários,

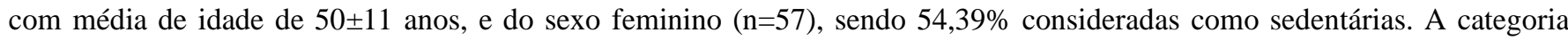
raça/cor preto, pardo ou outro prevaleceu, sendo 55,95\% no Grupo A e 44,05\% no Grupo B. Em relação ao status marital entre os sedentários, a maior parte dos sedentários referiu ter companheiro (casado/outra união) (60,87\%). Já a densidade familiar foi homogênea entre os grupos (Grupo A: 57,58\% e 58,33\% versus. Grupo B: 42,42\% e 41,67\%) (Tabela1).

A maioria dos participantes do presente estudo $(n=55)$ referiu baixo nível de escolaridade ( $\leq 4$ anos de estudo), com percentuais similares entre os grupos A e B $(49,09 \%$ e 50,91\%). Já a baixa renda se mostrou mais elevada dentre os sedentários, sendo da ordem de $57,69 \%$, versus $42,31 \%$ dentre aqueles não sedentários. Por sua vez, uma menor densidade domiciliar ( $\mathrm{n}=66)$ foi observada em ambos os grupos, porém com maior percentual dentre sedentários $(57,58 \%)$. Já o quantitativo de dois ou mais filhos se mostrou mais frequente $(\mathrm{n}=65)$, especialmente nos considerados sedentários $(55,38 \%)$ (TABELA1).

Por sua vez, o hábito de fumar e o consumo de bebidas alcoólicas se mostrou mais elevado dentre os sedentários, com frequências de 83,33\% e 58,62\%, respectivamente. Quanto à frequência de SM, segundo os critérios do NCEP-ATPIII, observou-se que $57,14 \%$ dos sedentários mostraram tal condição, versus $42,22 \%$ dos não sedentários (Tabela1). 
Tabela 1 - Características socioeconômico-demográficas e presença de SM da amostra de acordo com presença ou ausência de sedentarismo. Salvador, BA, Brasil $(n=90)$.

\begin{tabular}{|c|c|c|c|c|c|}
\hline \multirow[b]{2}{*}{ Características } & \multicolumn{4}{|c|}{ Sedentarismo } & \multirow[b]{3}{*}{$\mathbf{p}^{*}$} \\
\hline & \multicolumn{2}{|c|}{$\underset{(n=52)}{\operatorname{Sim}}$} & \multicolumn{2}{|c|}{$\begin{array}{c}\text { Não } \\
(\mathbf{n}=38)\end{array}$} & \\
\hline Idade (em anos) & $\mathbf{n}$ & $\%$ & $\mathbf{N}$ & $\%$ & \\
\hline$\leq 53$ & 32 & 66,67 & 16 & 33,33 & \multirow{2}{*}{0,068} \\
\hline$>53$ & 20 & 47,62 & 22 & 52,38 & \\
\hline Média $\pm d p$ & \multicolumn{2}{|c|}{$50 \pm 11$} & \multicolumn{2}{|c|}{$57 \pm 11$} & \\
\hline Mediana & \multicolumn{2}{|c|}{50,5} & \multicolumn{2}{|c|}{54,5} & \\
\hline Mínimo-Máximo & \multicolumn{2}{|c|}{$30-75$} & \multicolumn{2}{|c|}{$30-77$} & \\
\hline \multicolumn{6}{|l|}{ Sexo } \\
\hline Feminino & 31 & 54,39 & 26 & 45,61 & \multirow{2}{*}{0,392} \\
\hline Masculino & 12 & 36,36 & 21 & 63,64 & \\
\hline \multicolumn{6}{|l|}{ Raça/ cor da pele } \\
\hline Branco/Amarelo & 1 & 16,67 & 5 & 83,33 & \multirow{2}{*}{0,191} \\
\hline Preto/Pardo/Outro & 47 & 55,95 & 37 & 44,05 & \\
\hline \multicolumn{6}{|l|}{ Estado Civil } \\
\hline Casado/outra união & 28 & 60,87 & 18 & 39,13 & \multirow{2}{*}{0,544} \\
\hline Solteiro/Divorciado/Viúvo & 24 & 54,45 & 20 & 45,45 & \\
\hline \multicolumn{6}{|l|}{ Nível de Escolaridade (em anos) } \\
\hline$>4$ anos & 25 & 71,43 & 10 & 28,57 & \multirow{2}{*}{0,036} \\
\hline$\leq 4$ anos & 27 & 49,09 & 28 & 50,91 & \\
\hline \multicolumn{6}{|l|}{$\bar{R}$ enda familiar ${ }^{1}$} \\
\hline$\leq 1$ salário mínimo & 30 & 57,69 & 22 & 42,31 & \multirow{2}{*}{0,985} \\
\hline$>1$ salário mínimo & 22 & 57,89 & 16 & 42,11 & \\
\hline \multicolumn{6}{|l|}{$\begin{array}{l}\text { Densidade Domiciliar } \\
\text { (número de pessoas por domicílio) }\end{array}$} \\
\hline$\leq 3$ pessoas & 38 & 57,58 & 28 & 42,42 & \multirow{2}{*}{0,949} \\
\hline$>3$ pessoas & 14 & 58,33 & 10 & 41,67 & \\
\hline \multicolumn{6}{|l|}{ Número de filhos } \\
\hline$\leq 1$ filho & 16 & 64,00 & 9 & 36,00 & \multirow{2}{*}{0,459} \\
\hline$\geq 2$ filhos & 36 & 55,38 & 29 & 44,62 & \\
\hline \multicolumn{6}{|l|}{ Hábito de fumar atual } \\
\hline Sim & 5 & 83,33 & 1 & 16,67 & \\
\hline Não & 37 & 44,05 & 47 & 55,95 & 0,190 \\
\hline Consumo de bebida alcoólica & & & & & \\
\hline Sim & 17 & 58,62 & 12 & 41,38 & \\
\hline Não & 35 & 57,38 & 26 & 42,62 & 0,911 \\
\hline SM & & & & & \\
\hline Sim & 24 & 57,14 & 18 & 42,86 & \\
\hline$N \tilde{o} o$ & 28 & 58,33 & 20 & 41,67 & 0,909 \\
\hline
\end{tabular}

* Nível de significância estatística: $\mathrm{p} \leq 0,05$;

${ }^{1}$ Valor do salário mínimo $\mathrm{R} \$ 954,00$ na data da coleta dos dados.

Fonte: Autores.

Considerando as condições gerais de saúde, constatou-se um grande percentual de SM entre indivíduos hipertensos $(60,00 \%)$, diabéticos $(56,06 \%)$, portadores de doenças cardiovasculares (DCV) $(58,23 \%)$. Ainda em relação aos participantes com SM, 49,15\% estavam com alto IMC, indicando sobrepeso, e uma Relação Cintura-Quadril (RCQ) menor foi mais frequente na amostra (Tabela 2). 
Tabela 2 - Distribuição das características relacionadas com as condições gerais de saúde de acordo com diagnóstico da síndrome metabólica. Salvador, Bahia-Brasil (n=90).

\begin{tabular}{|c|c|c|c|c|c|}
\hline \multirow{3}{*}{ Características } & \multicolumn{4}{|c|}{ Diagnóstico SM (NCEP- ATPIII) } & \multirow[b]{3}{*}{$\mathbf{p}$} \\
\hline & \multicolumn{2}{|c|}{$\operatorname{Sim}(n=42)$} & \multicolumn{2}{|c|}{ Não $(n=48)$} & \\
\hline & $\mathbf{n}$ & & $\mathbf{n}$ & $\%$ & \\
\hline \multicolumn{5}{|l|}{ Horas de Sono } & \\
\hline$\geq 8$ horas & 24 & 66,67 & 12 & 33,33 & \multirow[t]{2}{*}{0,163} \\
\hline$<8$ horas & 28 & 51,85 & 26 & 48,15 & \\
\hline \multicolumn{6}{|l|}{ Hipertensão } \\
\hline Sim & 27 & 60.00 & 18 & 40.00 & \multirow{2}{*}{0,670} \\
\hline Não & 25 & 55.56 & 20 & 44.44 & \\
\hline \multicolumn{6}{|l|}{ Diabetes } \\
\hline Sim & 37 & 56,06 & 29 & 43,94 & \multirow{2}{*}{0,584} \\
\hline Não & 15 & 62,50 & 9 & 37,50 & \\
\hline \multicolumn{6}{|l|}{ Histórico de AVC } \\
\hline Sim & 50 & 56,82 & 38 & 43,18 & \multirow{2}{*}{0,221} \\
\hline Não & 2 & 100,00 & - & 0,00 & \\
\hline \multicolumn{6}{|l|}{ Doença Cardiovascular (DCV) } \\
\hline Sim & 46 & 58,23 & 33 & 41,77 & \multirow[b]{2}{*}{0,817} \\
\hline Não & 9 & 54,55 & 5 & 45,45 & \\
\hline \multicolumn{6}{|l|}{ Doença renal } \\
\hline Sim & 50 & 57,47 & 37 & 42,53 & \multirow{2}{*}{0,751} \\
\hline Não & 2 & 66,67 & 1 & 33,33 & \\
\hline \multicolumn{6}{|l|}{ Hipotireoidismo } \\
\hline $\operatorname{Sim}$ & 49 & 56,98 & 37 & 43,02 & \multirow{2}{*}{0,476} \\
\hline Não & 3 & 75,00 & 1 & 25,00 & \\
\hline \multicolumn{6}{|c|}{ Índice de Massa Corporal (peso/altura²) } \\
\hline$<25$ & 23 & 74,19 & 8 & 25,81 & \multirow{2}{*}{0,022} \\
\hline$\geq 25$ & 29 & 49,15 & 30 & 50,85 & \\
\hline \multicolumn{6}{|l|}{ Medida da cintura } \\
\hline$<93,24$ & 29 & 67,44 & 14 & 32,56 & \multirow{2}{*}{0,076} \\
\hline$\geq 93,24$ & 23 & 48,94 & 24 & 51,06 & \\
\hline \multicolumn{6}{|l|}{ Relação cintura quadril (RQC) } \\
\hline$\leq 0,87$ & 28 & 59,57 & 19 & 40,43 & \multirow{2}{*}{0,718} \\
\hline$>0,87$ & 24 & 55,81 & 19 & 44,19 & \\
\hline
\end{tabular}

* Nível de significância estatística: $\mathrm{p} \leq 0,05$

Fonte: Autores.

Quando se realizou a regressão logística, não foi detectado associação significante entre sedentarismo e Síndrome Metabólica, seja bruta $(\mathrm{OR}=0,95)$, ou ajustada por sexo, idade e uso de álcool $(\mathrm{OR}=1,02)$ (Tabela 3). 
Tabela 3 - Odds Ratio (OR) e Intervalo de Confiança (IC 95\%) obtidos mediante regressão logística não condicional para a associação sedentarismo e Síndrome Metabólica, Feira de Santana, Bahia, Brasil, 2020 (n=90).
SEDENTARISMO
MODELOS
OR
IC $95 \%$

\section{$\mathbf{p}^{*}$}

Bruto (n=90)

$(0,41-2,20)$

SÍNDROME

METABÓLICA
0,95

1,02
$(042-2,43)$

$\operatorname{Ajustado}^{1}(\mathrm{n}=90)$

\footnotetext{
${ }^{1}$ Ajustado por: sexo, idade, uso de álcool.

* Valor de significância estatística: $\mathrm{p} \leq 0,05$.

Fonte: Autores.
}

\section{Discussão}

A partir dos critérios do NCEP-ATP III, os presentes resultados indicam uma alta prevalência de SM na amostra estudada, com a maioria dos casos ocorrendo em mulheres. Taxas de prevalências de SM similares também foram descritas no Rio de Janeiro por Leão, Barros e Koifman (2010) e por Kubrusly, Oliveira e Simões (2015), que encontraram, respectivamente, taxas de 55,6\% e 41,7\%, diferentemente do que foi encontrado por Rodrigues et al. (2017), somente 19,2\% de prevalência de SM.

No que se refere à predominância de mulheres na presente amostra, achados similares foram apresentados por Lira Neto et al. (2018) em uma unidade de saúde no estado do Piauí. No entanto, é relevante considerar a possibilidade de efeitos específicos nesse grupo decorrentes da existência de determinantes biológicos que possibilitem a ocorrência da SM em adultos, e devido a maior adesão das mulheres em buscar atenção e serviços de saúde (Gottlieb, Cruz \& Bodanese, 2008). Nesse sentido, reafirma-se que a alta taxa de encaminhamento de usuários com obesidade para os serviços da atenção especializada é indicativa de que a Atenção Básica em Saúde não tem sido efetiva no tratamento e cuidado desses usuários (Bortolini et al., 2020). Isso suscita a necessidade de reflexões acerca da assistência prestada aos usuários como um todo, para além da amostra estudada.

Em relação aos aspectos socioeconômicos, a presente investigação detectou homogeneidade no que se refere à renda familiar dos participantes. Isso contradiz os achados que apontaram maior prevalência de SM em pessoas com menores condições financeiras (Salaroli, Barbosa \& Mill, 2007). No tocante à variável raça/cor, observou-se predomínio de pessoas que auto referiram-se como pretos, pardos ou outros, achado esse compatível com os resultados apresentados por Mendes, Theodoro \& Rodrigues (2012) em revisão sistemática publicada em 2019.

A SM por ser um grupo de distúrbios que inclui obesidade, resistência à insulina, dislipidemia e hipertensão, vem ganhando importância devido à sua associação com o desenvolvimento subsequente de doença cardiovascular e DM tipo 2. Ela envolve uma tolerância à glicose prejudicada, dentre outros fatores. Esse indicador pode estimular consequências de distúrbios metabólicos, que quando somados classificam a presença de SM (SBD, 2016; Brandão, Brandão \& Berenson, 2005).

No presente estudo, foi detectada uma elevada taxa de indivíduos com sobrepeso ou obesidade que foi encontrada $(65,55 \%)$. Resultado similar a este foi encontrado por Rodrigues et al. (2017) no valor de 53,9\%. Embora não tenha havido diferenças em relação a RCQ entre os grupos de comparação ora investigados, verificou-se maior prevalência de SM naqueles que apresentaram RCQ mais elevada $(55,81 \%)$.

Nesse contexto, é importante considerar o possível efeito do sedentarismo em tais indicadores, visto que a maior parte dos indivíduos ora investigados eram sedentários. Cabe ressaltar que no Brasil foi verificado em 2013 que o excesso de peso alcançava mais da metade dos brasileiros adultos, e a obesidade atingia 16,8\% dos homens e 24,4\% das mulheres (Ferreira, 
Szwarcwald \& Damacena, 2013). Além do que, foi demonstrado por Malta et al. (2019) um incremento gradual das taxas de obesidade, hipertensão arterial e diabetes na população adulta do Brasil.

Souza (2010) já chamava atenção para a necessidade de estratégias de saúde pública capazes de modificar padrões da atividade física, além do comportamento alimentar. No entanto, os dados ora encontrados apontam para uma ineficácia da atenção básica de saúde no grupo estudado em relação ao controle efetivo de tais condições.

Nesse sentido, reafirma- se o papel fundamental da prática da atividade física para o gasto energético e a manutenção do controle do peso, além de contribuir na redução da pressão arterial, elevação da taxa de HDL e melhor controle glicêmico.

A regularidade da atividade física atua na prevenção de doenças, evitando sua progressão e contribuindo com uma melhor qualidade de vida (Bernardes et al., 2018). Logo, é de importância singular na regulação de diversos fatores de risco cardiovasculares, tais como hipertensão, obesidade e dislipidemia (SBD, 2017). Segundo Liu, Lu \& Shi (2015), a atividade física é efetiva na redução do peso, além de ser um aliado no combate à SM e melhoria na qualidade de vida (Saboya, Bonadese \& Zimmermann, 2016). Nesse quesito, ressalta-se a necessidade de uma atuação intensa e eficaz dos profissionais de saúde da atenção básica para o estímulo à prática de atividade física e ao autocuidado. Isso envolve educação em saúde que tem relação direta com uma melhor adesão de usuários a boas práticas e saúde.

Por outro lado, deve também ser considerado que as modificações do padrão nutricional da população são confirmadas pelo aumento na taxa de indivíduos com sobrepeso e obesos, e a diminuição da desnutrição (Pinheiro, Freitas \& Corso, 2004). Este fato decorre dos atuais hábitos alimentares da população brasileira, como o aumento do consumo de alimentos industrializados, ricos em gorduras, açúcar simples e sódio, e pobres em fibras. Esses novos hábitos alimentares podem estar dentre os fatores principais responsáveis pelo elevado índice de indivíduos acima do peso ora detectado (MolenaFernandes, Nardo-Junior \& Tasca, 2005).

Dessa forma, segundo Nilson et al. (2020), devem ser fortalecidas medidas que possam se deter ao crescimento das DCNT, associadas a fatores dietéticos, incluindo a priorização de escolhas alimentares melhores. Merece destaque, a necessidade de estratégias mais assertivas de educação em saúde nos participantes desse estudo, quiçá de muitos outros usuários da unidade em questão, com vistas a redução do peso, além dos demais benefícios que dela possam decorrer, conforme acima citado.

Além do impacto social direto, é relevante considerar o impacto econômico associado a doenças e fatores de risco cardiometabólicos. No Brasil, os custos estimados de DCV chegaram a R \$ 37,1 bilhões no ano de 2015, incluindo os custos estimados por morte prematura, os custos diretos com internações e as perdas de produtividade relacionadas à doença (Siqueira, Siqueira-Filho \& Land, 2017). Também foi observado que os gastos hospitalares e ambulatoriais do SUS com obesidade são muito elevados, e chegaram a US\$269,6 milhões, sendo que 24\% deles foram atribuídos à obesidade mórbida (Oliveira, Santos \& Silva, 2015; Gonçalves \& Silva, 2018).

Sabe-se que hábitos alimentares saudáveis são indispensáveis na promoção da saúde cardiovascular, atuando, especialmente, nos níveis séricos de colesterol total e frações. O colesterol é necessário ao organismo, mas torna-se prejudicial em nível elevado, podendo ser um componente fatal para indivíduos com predisposição a eventos cardíacos como a aterosclerose. Seu excesso promove a formação de placas que obstruem as artérias, principalmente, o LDL, o colesterol ruim ou de baixa densidade, que se mostra associado ao excesso de peso e sedentarismo (Saboya et al., 2016; Bergmann, 2011; Sociedade Brasileira de Cardiologia [SBC], 2013; Sales, Peluzio \& Costa, 2003; Schaefer et al., 2001; Lusis, 2000).

A má nutrição e seus efeitos têm representado um ônus considerável aos sistemas de saúde. Nesse sentido, a Década de Ação sobre a Nutrição das Nações Unidas (2016-2025) estabeleceu a necessidade de enfrentamento de todas as formas de má nutrição, com destaque para desnutrição, carências nutricionais específicas, sobrepeso e obesidade e DCNT associadas à alimentação, inclusive hipertensão arterial e diabetes mellitus (United Nations General Assembly [UM], 2018). Merece 
ressalva o aumento verificado na venda e no consumo de alimentos ultra processados em diversas regiões, inclusive na América Latina, além do excesso de sódio, gorduras e açúcar nas dietas, configurando um desafio às políticas de saúde no combate as doenças crônicas (Organização Pan-Americana da Saúde [OPAS], 2018; Brasil, 2019).

Destaca-se a necessidade de priorizar ações para usuários com doenças crônicas, incluindo a estratificação do risco, estabilização da doença e aprimoramento do autocuidado, com ênfase para a alimentação e na atividade física. Logo, é suscitada a incorporação de tais iniciativas na rotina e nas práticas dos profissionais de saúde, centradas no diagnóstico nutricional, desenvolvimento de ações de promoção da alimentação adequada e saudável e de atividades físicas (Bortolini et al., 2020). Certamente medidas dessa natureza poderão ter um impacto positivo no grupo ora investigado.

A da amostra não ter tido poder para detectar a possível uma associação significante entre sedentarismo e SM, uma tendência de efeito foi observada na medida ajustada obtida $(\mathrm{OR}=1,02)$. Certamente, o baixo poder da amostra não permitiu avaliar o real efeito do sedentarismo no desfecho em tela. Isso suscita pesquisas mais amplas e empoderadas, para ser possível obter fundamentos mais consubstanciados e com mais capacidade de balizar reorganização de estratégias e influenciar novas políticas de saúde para enfrentamento das doenças cardiometabólicas. No entanto, reafirma-se que, frente a maior tendência de consumo de alimentos mais calóricos, a reversão do sedentarismo é fundamental não somente para a prevenção ou controle da SM, mas para a saúde como um todo.

Além disso, Sharma et al. (2019) chamam atenção para uma outra consequência negativa, que se refere à possível barreira para a busca do serviço de saúde pelos usuários para tratamento da obesidade representada pelo estigma e o preconceito que tais indivíduos possam sofrer devido ao seu peso.

\section{Considerações Finais}

Sabe-se que a prevalência da SM é diretamente relacionada ao excesso de peso, principalmente com a obesidade abdominal, independentemente dos critérios utilizados para seu diagnóstico. Ainda, é importante destacar que mudanças no estilo de vida, como adoção de hábitos alimentares saudáveis e práticas regulares de atividade física que promovem a perda de peso, podem acarretar a diminuição da SM.

Nesse contexto, ressalva deve ser feita ao papel da atenção básica enquanto porta de entrada do sistema público de saúde brasileiro, na promoção da saúde nutricional e metabólica, bem como na prevenção de condições que configuram estilo não saudáveis de vida, a exemplo do sedentarismo.

Embora os presentes resultados não tenham demonstrado um efeito independente do sedentarismo na ocorrência da SM, as altas taxas de fatores de risco cardiometabólicos identificados na amostra, convidam para reflexões quanto à efetividade de políticas e saúde voltadas para sua prevenção. Da mesma forma, apontam para a necessidade de qualificação dos profissionais da APS, no tocante às orientações sobre a importância da atividade física regular e da alimentação adequada e saudável, além do acolhimento assertivo às pessoas com excesso de peso e/ou obesidade.

Além disso, apontam para a necessidade de realização de novas pesquisas com maior robustez amostral, em especial, estudos de acompanhamento, tipo coorte e de intervenção. Isso para obter uma melhor compreensão da cadeia de causalidade da SM, considerando seus fatores de risco, suas implicações e quais grupos estariam em maior risco, além do seu impacto amplo à saúde humana.

\section{Referências}

Bergmann, M. L de A., Bergmann, G. G., Halpern, R., Rech, R. R., Constanzi, C. B, \& Alli, L. R. (2011). Colesterol total e fatores associados: estudo de base escolar no sul do Brasil. Arquivos Brasileiros de Cardiologia, 97(1), 17-25. https://doi.org/10.1590/S0066-782X2011005000065 
Bernardes, N. D, da S. D., Stoyell-Conti, F. F., Brito-Monzani, J. de O., Malfitano, C., Caldini, E. G., Ulloa, L., Llesuy, S. F., Irigoyen, M-C, \& De Angelis, K. (2018). Baroreflex Impairment Precedes Cardiometabolic Dysfunction in an Experimental Model of Metabolic Syndrome: Role of Inflammation and Oxidative Stress. Scientific reports, 8(1), 8578. https://doi.org/10.1038/s41598-018-26816-4

Bonomini, F., Rodella, L. F., Rezzani, R. (2015). Metabolic syndrome, aging and involvement of oxidative stress. Aging Dis. Mar 10;6(2):109-20. 10.14336/AD.2014.0305.

Bortolini, G. A., Oliveira, T., Silva, S. A., Santin, R., Medeiros, O. L., Spaniol, A. M., Pires, A., Alves, M, \& Faller, L. A. (2020). Ações de alimentação e nutrição na atenção primária à saúde no Brasil [Feeding and nutrition efforts in the context of primary healthcare in BrazilMedidas relativas a la alimentación y la nutrición en la atención primaria de salud en Brasil]. Revista panamericana de salud publica = Pan American journal of public health, 44, e39. https://doi.org/10.26633/RPSP.2020.39

Brandão, A. P., Brandão, A. A., Berenson, G. S. (2005). Síndrome Metabólica em Crianças e Adolescentes. Arquivos Brasileiros de Cardiologia, 85(2), 79-81. https://doi.org/10.1590/S0066-782X2005001500001

Brasil. Ministério da Saúde (2001). Secretaria de Políticas de Saúde. Departamento de Ações Programáticas Estratégicas. Plano de reorganização da atenção à hipertensão arterial e ao diabetes mellitus: hipertensão arterial e diabetes mellitus / Departamento de Ações Programáticas Estratégicas. - Brasília: Ministério da Saúde, 2001. 102 p.: il. - (Série C. Projetos, Programas e Relatórios; n. 59).

Brasil. Ministério da Saúde (2018). Síndrome Metabólica. Biblioteca Virtual em Saúde. 15 de Fevereiro de 2018. http://bvsms.saude.gov.br/bvs/publicacoes/reorganizacao_plano.pdf.

Brasil. Ministério da Saúde. Saúde Brasil (2019). Uma análise da situação de saúde e das doenças e agravos crônicos: desafios e perspectivas. Brasília: Secretaria de Vigilância em Saúde. http://bvsms.saude.gov.br/bvs/publicacoes/saude_brasil_2018_analise_situacao_saude_doencas_agravos_cr onicos_desafios_perspectivas.pdf

Ferreira, A. P. de S., Szwarcwald, C. L. \& Damacena, G. N. (2013). Prevalência e fatores associados da obesidade na população brasileira: estudo com dados aferidos da Pesquisa Nacional de Saúde. Revista Brasileira de Epidemiologia, 22, e190024. https://doi.org/10.1590/1980-549720190024

França, M. L., Souza, S. S., Marques, N. da S. F. (2017). Benefícios da prática de exercício físico em pacientes com síndrome metabólica. DêCiência em Foco; 1(1), 30-47.

Gomes-Filho, I. S, das Mercês, M. C., de Santana, P. S. J., Seixas, da Cruz S., Teixeira, L. A. M., Trindade, S. C, de Moraes, M. C. E., Freitas, C. J. M., Marques M. F. M., Barreto, M. L., Pereira, V. M. I., Nascimento, C. M. C., Seymour. G. J., \& Scannapieco, F. A (2016). Severity of Periodontitis and Metabolic Syndrome: Is There an Association? J Periodontol. 87(4), 357-66. 10.1902/jop.2015.150367.

Goncalves, G. M. R., \& Silva, E. N. D. (2018). Cost of chronic kidney disease attributable to diabetes from the perspective of the Brazilian Unified Health System. PLoS One. 13(10):e0203992. 10.1371/journal.pone.0203992.

Gottlieb, M. G. V., Cruz, I. B. M., \& Bodanese, L. C. (2008). Origem da síndrome metabólica: aspectos genético-evolutivos e nutricionais. Sci Med. 18(1):318.

Han T. S., \& Lean M. E. J. (2010). Metabolic syndrome. Medicine. 39:24-31.doi.org/10.1016/j.mpmed.2010.10.010.

Kassi, E., Pervanidou, P., Kaltsas, G., \& Chrousos, G. (2011). Metabolic syndrome: definitions and controversies. BMC medicine, 9 , 48. https://doi.org/10.1186/1741-7015-9-48

Kubrusly, M., Oliveira, C. M. C., Simões, P. S. F., Lima, R. D. O., Galdino, P. N. R., Sousa, P. D. A. F., Jerônimo, A. L. C. (2015). Prevalência de síndrome metabólica diagnosticada pelos critérios NCEP-ATP III e IDF em pacientes em hemodiálise. J Bras Nefrol. 37(1):72-8. https://doi.org/10.5935/01012800.20150011 .

Leão, L. S. C. S., Barros, E. G., Koifman, R. J. (2010). Prevalência de síndrome metabólica em adultos referenciados para ambulatório de nutrição no Rio de Janeiro, Brasil. Rev Bras Cardiol. 23(2):93-100.

Lira Neto, J. C. G., Oliveira, J. F. S. F., Souza, M. A, Araújo, M. F. M., Damasceno, M. M. C., Freitas, R. W. J. F. (2018). Prevalência Da Síndrome Metabólica e de Seus Componentes em Pessoas com Diabetes Mellitus Tipo 2. Texto Contexto Enferm. 27(3):1-8, e3900016. https://www.scielo.br/pdf/tce/v27n3/0104-0707-tce-27-03-e3900016.pdf.

Liu, Y. L., Lu, C. W., Shi, L., Liou, Y. M., Lee, L. T., Huang, K. C. (2015). Low intensive lifestyle modification in young adults with metabolic syndrome a community-based interventional study in Taiwan. Medicine (Baltimore).94(22):e916. 10.1097/MD.0000000000000916.

Lusis, A. J. (2000). Atherosclerosis. Nature. 407(6801):233-41. 10.1038/35025203.

Malta, D. C., Saltarelli, R. M. F., Prado, R. R. M., Rosane, A., \& Almeida, M. F. (2018). Mortes evitáveis no Sistema Único de Saúde na população brasileira, entre 5 e 69 anos, 2000 - 2013. Revista Brasileira de Epidemiologia, 21, e180008. https://doi.org/10.1590/1980-549720180008

Malta, D. C., Duncan, B. B., Schmidt, M. I., Machado, Í. E., Silva, A. G., Bernal, R. T. I., Pereira, C. A., Damacena, G. N., Stopa, S, R., Rosenfeld, L. G., \& Szwarcwald, C. L. (2019). Prevalência de diabetes mellitus determinada pela hemoglobina glicada na população adulta brasileira, Pesquisa Nacional de Saúde. Revista Brasileira de Epidemiologia, 22(Suppl. 2), E190006.SUPL.2. https://doi.org/10.1590/1980-549720190006.supl.2

Mendes, K. G., Theodoro, H., Rodrigues, A. D., \& Olinto, M. T. A. (2012). Prevalência de síndrome metabólica e seus componentes na transição menopáusica: uma revisão sistemática. Cadernos de Saúde Pública, 28(8), 1423-1437. https://doi.org/10.1590/S0102-311X2012000800002

Molena-Fernandes, C. A., Nardo Junior, N., Tasca, R. S., Cuman, R. K. N (2005). Importância da associação de dieta e de atividade física na prevenção e controle do Diabetes Mellitus tipo 2. Acta Scientiarum. Health Science, Maringá, 27(2): 195-205. 
National Cholesterol Education Program's Adul Treatmen Panel III (2001). Expert Panel on Detection, Evaluation, and Treatment of High Blood Cholesterol in Adults. Executive Summary of The Third Report of The National Cholesterol Education Program (NCEP) Expert Panel on Detection, Evaluation, And Treatment of High Blood Cholesterol In Adults (Adult Treatment Panel III). 285(19):2486-9710.1001/jama.285.19.2486.

Nilson, E., Andrade, R., de Brito, D. A., \& de Oliveira, M. L. (2020). Custos atribuíveis a obesidade, hipertensão e diabetes no Sistema Único de Saúde, Brasil, 2018 [Costs attributable to obesity, hypertension, and diabetes in the Unified Health System, Brazil, 2018 Costos atribuibles a la obesidad, la hipertensión y la diabetes en el Sistema Único de Salud de Brasil, 2018]. Revista panamericana de salud publica = Pan American journal of public health, 44, e32. https://doi.org/10.26633/RPSP.2020.32

Ponte

Ponte, G. A., Silva, C. S., Dantas, Bruna Matos Santos, Galvão, L. R., Galvão, C. R., Santos Neto, E. S., Portugal, G. A., Damasceno, K. S. M., Mercês, M. C. das, Coelho, J. M. F. (2020). Periodontite e síndrome metabólica: diferenças entre critérios de diagnóstico e sexo. Research, Society and Development, 9(9) e265997265-27.

Oliveira, M. L., Santos, L. M., \& da Silva, E. N. (2015). Direct healthcare cost of obesity in brazil: an application of the cost-of-illness method from the perspective of the public health system in 2011. PloS one, 10(4), e0121160. https://doi.org/10.1371/journal.pone.0121160

Organização Pan-Americana da Saúde. Alimentos e bebidas ultraprocessados na América Latina: tendências, efeito na obesidade e implicações para políticas públicas. Brasília, DF: OPAS; 2018. https://www.cfn.org.br/wp-content/uploads/2018/05/Alimentos-e-bebidas-ultraprocessados-na-Am\%C3\%A9rica-Latina01.pdf

Ortega, F. B, Konstabel K., Pasquali, E., Ruiz, J. R., Hurtig-Wennlöf, A., Mäestu, J., Löf, M., Harro, J., Bellocco, R., Labayen, I., Veidebaum, T., Sjöström, M. Objectively measured physical activity and sedentary time during childhood, adolescence and young adulthood: a cohort study. PLoS One. 8(4):e60871 10.1371/journal.pone.0060871.

Pereira, A. S., Shitsuka, D. M., Parreira, F. J., Shitsuka, R. Metodologia da pesquisa científica. UFSM. https://repositorio.ufsm.br/bitstream/handle/1/15824/Lic_Computacao_Metodologia-Pesquisa-Cientifica.pdf?sequence=1

Pinheiro, A. R. de Oliveira, Freitas, S. F. T., \& Corso, A. C. T. (2004). Uma abordagem epidemiológica da obesidade. Revista de Nutrição, 17(4), 523-533. https://doi.org/10.1590/S1415-52732004000400012

Ramires, E. K. N., Mendonça, M., Risia, C. E. de, Longo-Silva, Giovana, S., Taíse G., Marinho, P. de Menezes, \& Silveira, J. A. C. (2018). Prevalence and Factors Associated with Metabolic Syndrome among Brazilian Adult Population: National Health Survey - 2013. Arquivos Brasileiros de Cardiologia, 110(5), 455-466. https://doi.org/10.5935/abc.20180072

Rodrigues, M. C, Silva, E. M, Silva, B. K. R, Quaresma, F. R. P, Sesti, L. F. C, Adami, F., Maciel, E. da S. (2017). Avaliação de fatores de risco para síndrome metabólica em grupo de funcionários de uma Escola Pública. Saúde em Revista, Piracicaba, 17(47), 11-22.

Saboya, P. P., Bodanese, L. C., Zimmermann, P. R., Gustavo, A., Assumpção, C. M., \& Londero, F. (2016). Síndrome metabólica e qualidade de vida: uma revisão sistemática. Revista Latino-Americana de Enfermagem, 24, e2848. .https://doi.org/10.1590/1518-8345.1573.2848

Salaroli, L. B., Barbosa, G C., Mill, J. G., \& Molina, M. C.B. (2007). Prevalência de síndrome metabólica em estudo de base populacional, Vitória, ES Brasil. Arquivos Brasileiros de Endocrinologia \& Metabologia, 51(7), 1143-1152. https://doi.org/10.1590/S0004-27302007000700018.

Sales, R. L, Peluzio, M. C. G., \& Costa, N. M. B. (2003). Lipoproteínas: uma revisão do seu metabolismo e envolvimento com o desenvolvimento de doenças cardiovasculares. Nutrire Rev. Soc. Bras. Aliment. Nutr. 25:71-86.

Santos, G. de O., Guimarães, A. H. A., Santos, L. M., \& Silva, S. L. (2020). A influência da prática de musculação na composição corporal e relação cintura quadril. Research, Society and Development, 9(10), e3669108782. http://dx.doi.org/10.33448/rsd-v9i10.8782

Schaefer, E. J., Audelin, M. C., McNamara, J. R., Shah, P. K., Tayler, T., Daly, J. A., Augustin, J. L., Seman, L. J., \& Rubenstein, J. J. (2001). Comparison of fasting and postprandial plasma lipoproteins in subjects with and without coronary heart disease. The American journal of cardiology, 88(10), 1129-1133. https://doi.org/10.1016/s0002-9149(01)02047-1

Silva, H. R. da, nascimento, F. R. dos S., santos, S. L. dos, Lustosa, M. J. L., Melo Filho, J. C. L. C. de, Portela, C. L., Costa, R. H. F., Macedo Júnior, C. A. A. de, Fernandes, L. K. da S., Pinheiro Neto, J. C. (2020). A importância da prática de atividades físicas e uma alimentação saudável na profilaxia de um câncer. Research, Society and Development, 9(4), e68942868. http://dx.doi.org/10.33448/rsd-v9i42868

Sociedade Brasileira de Cardiologia (2013). III Diretrizes Brasileiras Sobre Dislipidemias e Diretriz de Prevenção da Aterosclerose do Departamento de Aterosclerose da Sociedade Brasileira de Cardiologia. Arq. Bras. Cardiol. 77 (supl III): 1-48.

Sociedade Brasileira de Diabetes (2016). Diretrizes da Sociedade Brasileira de Diabetes (2015-2016) / Adolfo Milech...[et. al.]; organização José Egidio Paulo de Oliveira, Sérgio Vencio - São Paulo: A.C. Farmacêutica.

Sociedade Brasileira de Diabetes (2017). Diretrizes da Sociedade Brasileira de Diabetes 2017-2018. Organização José Egídio Paulo de Oliveira, Renan Magalhães Montenegro Junior, Sérgio Vencio. São Paulo: Editora Clannad. https://www.diabetes.org.br/profissionais/ images/2017/ diretrizes/diretrizes-sbd2017-2018.pdf.

Sharma, A. M., Bélanger, A., Carson, V., Krah, J., Langlois, M. F., Lawlor, D., Lepage, S., Liu, A., Macklin, D. A., MacKay, N., Pakseresht, A., Pedersen, S. D., Ramos Salas, X., \& Vallis, M. (2019). Perceptions of barriers to effective obesity management in Canada: Results from the ACTION study. Clinical obesity, 9(5), e12329. https://doi.org/10.1111/cob.12329

Shin, J. A., Lee, J. H., Lim, S. Y., Ha, H. S., Kwon, H. S., Park, Y. M., Lee, W. C., Kang, M. I., Yim, H. W., Yoon, K. H., \& Son, H. Y. (2013). Metabolic syndrome as a predictor of type 2 diabetes, and its clinical interpretations and usefulness. Journal of diabetes investigation, 4(4), 334-343. https://doi.org/10.1111/jdi.12075 
Research, Society and Development, v. 10, n. 1, e56910112195, 2021

(CC BY 4.0) | ISSN 2525-3409 | DOI: http://dx.doi.org/10.33448/rsd-v10i1.12195

Silva, C. S, Santos, B. M, Almeida, H. B. O., El Fahl, L. R., Galvão, L. R., Galvão, C. R., Porto, E. C. L., Miranda, S. S., Figueredo, A. C. M. G., Coelho, J. M. F. (2017). Estilo de vida e condição metabólica de mulheres diabéticas e/ou hipertensas de uma região urbana. Rev. Saúde Col. UEFS, 7(2): 65-72. http://periodicos.uefs.br/index.php/saudecoletiva/article/ view/1395

Siqueira, A. S. E., Siqueira-Filho, A. G., Land, M. G. P. (2017). Analysis of the Economic Impact of Cardiovascular Diseases in the Last Five Years in Brazil. Arq Bras Cardiol. 109(1):39-46. https://doi.org/10.5935/abc.20170068.

Souza, E. B. (2010). Transição nutricional no Brasil: análise dos principais fatores. Cadernos Uni FOA. 13:49-53. http://web.unifoa.edu.br/ cadernos/edicao/13/49.pdf

United Nations General Assembly. United Nations Decade of Action on Nutrition (2016-2025). Work Programme. https://www.un.org/nutrition/un-decadeaction-nutrition-2016-2025. 